\title{
Therapie von Spondyloarthritiden - welches ist das beste Medikament im Land?
}

Die Frage nach dem besten Medikament hielt Prof. Dr. Filip van den Bosch, Gent, Belgien, in der Howto-Treat-Sitzung zu Spondyloarthritiden für nicht zielführend. Stattdessen solle sich der Arzt eine Reihe von Strategiefragen stellen. Denn letztlich könnten alle verfügbaren Medikamente mit Erfolg eingesetzt werden.

Aus Sicht von van den Bosch, Leiter der Klinik für Rheumatologie am Universitätsklinikum der Universität Gent, haben Strategien für eine frühe und anhaltende Remission einer Spondyloarthritis - sei es eine PsoriasisArthritis (PsA) oder eine axiale Spondyloarthritis (axSpA) - nichts damit zu tun, basierend auf Zulassungsstudien, ein besonderes Medikament auszusuchen. Dies gelte zumal dann, wenn darin nur etwa ein Viertel der Patienten das hochgesteckte Ziel einer inaktiven Erkrankung gemäß ASDAS (Ankylosing Spondylitis Disease Activity Score, ASDAS-IR, < 1,3) erreicht hätte, so van den Bosch beim virtuellen Kongress der European League Against Rheumatism (EULAR) 2021.

Stattdessen plädierte er dafür, sich an folgende vier klinische Grundsätze zu halten: Korrekte Diagnose der zugrundliegenden Erkrankung anhand der Zeichen und Symptome des Patienten. Korrekte Einschätzung der Beziehung zwischen den Zeichen und Symptomen und der ursächlichen Pathophysiologie. Behandlung modifizierbarer patientenindividuellen Faktoren, da beispielsweise Ängste und Depression einer effektiven Therapie im Wege stehen und zu einer schnellen und unreflektierten Eskalation der Therapie führen können. Und: Wahl des passgenauen Medikamentes aus dem Sortiment vieler guter Medikamente. Tendenziell sollten bei starker Hautbeteiligung eher Interleukin 17- und 23-Inhibitoren eingesetzt werden, bei chronisch-entzündlichen Darmerkrankungen erstere dagegen eher nicht.

\section{Fünf erfolgreiche Strategien}

Zusätzlich zu diesen vier Grundsätzen hält sich van den Bosch in der Behandlung von SpA-Patienten an fünf Strategien, die er ebenfalls zur Nachahmung empfahl:

- Strategie 1: Klassiker nicht vergessen und korrekt einsetzen. Denn auch ältere Medikamente wie Naproxen, Etoricoxib oder Methotrexat seien effektiv, auch in Kombination mit neuen zielgerichteten Medikamenten.

- Strategie 2: Effizienz der erstklassigen neuen Medikamente nutzen. Die heute verwendeten anspruchsvollen kombinierten Endpunkte werden unter den modernen zielgerichteten Therapien von vielen PsA-Patienten erreicht. Wie alles, haben die kombinierten Endpunkte aber ihre Vor- und Nachteile (z. B. Depression als Confounder für Gelenkendpunkte). Bei der axSpA sei es schwerer, über die Zeit Remissionsraten zu vergleichen, weil sich die Scores geändert hätten und nur heutige Studien Auskunft zur nichtröntgenologischen Form geben. Insbesondere mit Upadacitinib, seien nun höhere Remissionsraten möglich als bisher gewohnt.

- Strategie 3: Treat-to-Target und Tight Control. Den Nutzen dieser Strategien aus frühem Therapiebeginn, klarem Therapieziel und engmaschigen Kontrollen hätten die Studien TiCoPA für die PsA und TiCoSPA für die axSpA im Sinne eines besseren Therapieansprechens gezeigt.
- Strategie 4: Die

„DEER“-Behandlungshaltung des Arztes, wobei „DEER“ für Dedicated Empathic and Educated Rheumatologist steht. So kämen u.a. mentale und sozioökonomische Aspekte ans Licht, die ein Faktor für das Nicht-Ansprechen sein können.

- Strategie 5: Remissions-Induktions-Ansatz. Inzwischen hätten auch Studien zur SpA gezeigt, dass eine Strategie mit früher Induktion einer Remission nicht nur zu höheren Remissionsraten führt, sondern sich auch langfristig im Sinne einer anhaltenden Remission auszahlt, die wiederum ein Ausschleichen der Therapie bzw. eine Dosisreduktion mit gegebenenfalls Wiederaufnahme der Therapie bei einem Flare erlaubt. Als Beispiele nannte er die Studien CRESPA und SPIRIT P3 für die PsA sowie Re-EMBARK und C-OPTIMISE für die axSpA. Zusammengenommen könne so das beste Outcome für die Patienten erzielt werden.

Hinweis des Verlags. Der Verlag bleibt in Hinblick auf geografische Zuordnungen und Gebietsbezeichnungen in veröffentlichten Karten und Institutsadressen neutral.

rheuma plus $2021 \cdot 20: 199$

https://doi.org/10.1007/s12688-02100453-8

๑) Springer-Verlag GmbH Austria, ein Teil von Springer Nature 2021
Quelle: Session: How to treat spondyloarthritis, EULARKongress, virtuell, 2.-05.06.2021, Dr. Wiebke Kathmann, SpringerMedizin.de 